\title{
ThCOL2 Improves the Salt Stress Tolerance of Tamarix hispida
}

\author{
Xiaojin Lei, Bing Tan, Zhongyuan Liu, Jing Wu, Jiaxin Lv and Caiqiu Gao* \\ State Key Laboratory of Tree Genetics and Breeding, Northeast Forestry University, Harbin, China
}

The CONSTANS-LIKE (COL) transcription factor has been reported to play important roles in regulating plant flowering and the response to abiotic stress. To clone and screen $\mathrm{COL}$ genes with excellent salt tolerance from the woody halophyte Tamarix hispida, 8 ThCOL genes were identified in this study. The expression patterns of these genes under different abiotic stresses (high salt, osmotic, and heavy metal) and abscisic acid (ABA) treatment were detected using quantitative real-time PCR (qRT-PCR). The expression levels of 8 ThCOL genes changed significantly after exposure to one or more stresses, indicating that these genes were all stress-responsive genes and may be involved in the stress resistance response of $T$. hispida. In particular, the expression level of ThCOL2 changed significantly at most time points in the roots and leaves of

OPEN ACCESS

Edited by:

Ayako N. Sakamoto,

National Institutes for Quantum and Radiological Science and Technology, Japan

Reviewed by:

Tse-Min Lee,

National Sun Yat-sen University,

Taiwan

Nobuhiro Suzuki,

Sophia University, Japan

*Correspondence:

Caiqiu Gao

gaocaiqiu@nefu.edu.cn

Specialty section: This article was submitted to

Plant Abiotic Stress, a section of the journal Frontiers in Plant Science

Received: 15 January 2021 Accepted: 09 April 2021 Published: 17 May 2021

Citation:

Lei X, Tan B, Liu Z, Wu J, Lv J and

Gao $C$ (2021) ThCOL2 Improves the Salt Stress Tolerance of Tamarix hispida. Front. Plant Sci. 12:653791. doi: 10.3389/fpls.2021.653791
T. hispida under salt stress and after ABA treatments, which may play an important role in the response process of salt stress through a mechanism dependent on the ABA pathway. The recombinant vectors pROKII-ThCOL2 and pFGC5941-ThCOL2 were constructed for the transient transformation of T. hispida, and the transient infection of $T$. hispida with the pROKII empty vector was used as the control to further verify whether the ThCOL2 gene was involved in the regulation of the salt tolerance response of T. hispida. Overexpression of the ThCOL2 gene in plants under $150 \mathrm{mM} \mathrm{NaCl}$ stress increased the ability of transgenic $T$. hispida cells to remove reactive oxygen species (ROS) by regulating the activity of protective enzymes and promoting a decrease in the accumulation of $\mathrm{O}^{2-}$ and $\mathrm{H}_{2} \mathrm{O}_{2}$, thereby reducing cell damage or cell death and enhancing salt tolerance. The ThCOL2 gene may be a candidate gene associated with excellent salt tolerance. Furthermore, the expression levels of some genes related to the ABA pathway were analyzed using qRT-PCR. The results showed that the expressions of ThNCED1 and ThNCED4 were significantly higher, and the expressions of ThNCED3, ThZEP, and ThAAO3 were not significantly altered in OE compared with CON under normal conditions. But after $24 \mathrm{~h}$ of salt stress, the expressions of all five studied genes all were lower than the normal condition. In the future, the downstream genes directly regulated by the ThCOL2 transcription factor will be searched and identified to analyze the salt tolerance regulatory network of ThCOL2.

Keywords: Tamarix hispida, COL transcription factor, ThCOL2 gene, salt stress, ROS-scavenging capability

Abbreviations: NBT, nitrotetrazolium blue chloride; DAB, diaminobenzidine; SOD, superoxide dismutase; CAT, catalase; POD, peroxidase; MDA, malondialdehyde; FT, LOCUS T; Ghd7, grain height date 7; Ehd1, early heading date 1; OsRACK1, receptor for activated C-kinase 1; COL, CONSTANS-LIKE; Ghd2, grain height date 2. 


\section{INTRODUCTION}

The CONSTANS (CO) gene was first isolated in the Arabidopsis flowering delay mutant, and then 16 CONSTANS-LIKE (COL) genes were isolated in Arabidopsis, and together, these genes constitute a COL transcription factor family. The COL protein encoded by the $C O L$ gene contains two homologous domains: the B-box domain (zinc finger domain) located near the $\mathrm{N}$-terminus and the CCT (CO, CO-like, and TOC1) domain located near the $\mathrm{C}$-terminus. The COL family is divided into three groups based on the B-box domain. The first group contains two $\mathrm{B}$-box domains and is further subdivided into five subcategories according to the conserved region between the B-box and CCT domains: Ia, Ib, Ic, Id, and Ie. The second group contains one B-box domain. The third group contains one B-box domain and one zinc finger protein domain with changes in the secondary structure (Putterill et al., 1995; Robson et al., 2001).

In recent years, COL transcription factors have been identified in many plants, such as Arabidopsis thaliana (Zhang et al., 2014), Brassica juncea (Muntha et al., 2019), Phyllostachys violascens (Xiao et al., 2018), and Oryza sativa (Sheng et al., 2016). Most COL transcription factors have been reported to be involved in flowering regulation. In Arabidopsis, an increase in the expression of AtCOL4 leads to a decrease in the expression of $F T$ and APETALA 1 (AP1) and subsequently suppresses flowering (Steinbach, 2019). AtCOL3 inhibits flowering by interacting with $B B X 32$ to inhibit the transcription of the target gene $F T$ (Tripathi et al., 2017). In rice, OsCOL15 inhibits flowering by promoting Ghd7 expression and inhibiting RID1 expression (Wu et al., 2018). OsCOL10 inhibits flowering by reducing Ehd1 expression and acts on Ghd7 (Tan et al., 2016, 2017). OsCOL9 delays flowering time in rice by inhibiting the Ehd1 pathway (Liu et al., 2016a).

In addition to the regulation of flowering time, some $C O L$ genes are also involved in the regulation of plant responses to abiotic stress. Arabidopsis $O M G 1$ plays a role in regulating the ROS pathway by regulating related genes (MYB77 and GRX480) (Sng et al., 2018). AtCOL4 participates in salt stress responses through ABA-dependent signaling pathways (Min et al., 2015). Many $Z m C O L s$ are activated or inhibited to varying degrees in maize under $\mathrm{ABA}$ stress, indicating that $\mathrm{ZmCOLs}$ may be involved in stress responses related to the ABA pathway (Song et al., 2018). In rice, overexpression of the Ghd2 gene increases sensitivity to drought (Liu et al., 2016b). In banana, the expression of the MaCOL1 gene is significantly induced at different times of cold stress and participates in cold stress responses (Chen et al., 2012).

Moreover, the $C O L$ genes are also involved in other regulatory mechanisms. In apple (Malus domestica), the overexpression of $M d B B X 1$ reduces the ethylene content in transgenic apple strains and changes the anthocyanin concentration and expression of related genes to regulate fruit color (Plunkett et al., 2019). Rice OsCOL9 interacts with OsRACK1 and enhances rice blast resistance through salicylic acid and ethylene signaling pathways (Liu et al., 2016c). Morita et al. (2015) showed that the rice COL protein CRCT is a positive regulator of starch accumulation in vegetative tissues and regulates the coordinated expression of starch synthesis genes in response to photosynthesis. In Arabidopsis, AtCOL7 regulates the branching and shading response (Wang et al., 2013).

Tamarix hispida is a woody halophyte with strong salt tolerance. It grows normally in soil with less than $1 \%$ salt content. Therefore, it is an ideal material for studying the mechanism of salt tolerance and cloning salt tolerance genes (Gao et al., 2012). In the present study, 8 COL genes (named ThCOL1-8) were cloned from T. hispida, and their expression patterns in response to salt, osmotic, and heavy metal stresses and $\mathrm{ABA}$ treatments were analyzed in different T. hispida tissues. Furthermore, ThCOL2 was selected for further study and transiently transformed into T. hispida. ThCOL2 overexpression significantly improved the salt tolerance of transgenic T. hispida. This study provides a potential candidate salt tolerance-related gene for tree forestry genetics and breeding.

\section{MATERIALS AND METHODS}

\section{Plant Materials and Stress Treatments}

The culture conditions of T. hispida seedlings were determined according to the methods described by Wang et al. (2019). 2-months-old seedlings were exposed to one of the following solutions for $6,12,24,48$ or 72 h: $0.4 \mathrm{M} \mathrm{NaCl}, 20 \%$ (w/v) $\mathrm{PEG}_{6000}, 150 \mu \mathrm{M} \mathrm{CdCl}_{2}$ or $100 \mu \mathrm{M} \mathrm{ABA}$. At the same time, seedlings irrigated with fresh water were used as controls. After these treatments, the leaves and roots of at least 24 seedlings were harvested for RNA extraction. Three independent treatments were performed.

\section{Identification of the ThCOL Genes in T. hispida}

Eight ThCOL genes were identified from the seven transcriptome libraries (Wang et al., 2014) and confirmed based on a blast search of the conserved domain ${ }^{1}$. Then, the ThCOL genes with complete open reading frames (ORFs) were selected using ORF finder ${ }^{2}$. The GenBank accession numbers of ThCOL1-8 were MW846245846252. The molecular weight and theoretical isoelectric point (pI) of the ThCOL proteins were determined using ProtParam ${ }^{3}$.

\section{Bioinformatics Analysis of the ThCOL Genes}

The amino acid sequences of the 8 ThCOL proteins were selected for multiple sequence alignment, and 8 ThCOL proteins and 17 COL proteins in Arabidopsis were selected for the phylogenetic analysis. Multiple sequence alignments were performed using BioEdit software, and an unrooted phylogenetic tree was constructed with MEGA 5.05 software (Tamura et al., 2011).

\section{RNA Extraction and qRT-PCR}

Total RNA was extracted using the Plant total RNA extraction kit (BioTeke Corporation). According to the instructions from

\footnotetext{
${ }^{1}$ www.blast.ncbi.nlm.nih.gov/Blast.cgi

${ }^{2}$ https://www.ncbi.nlm.nih.gov/orffinder/

${ }^{3}$ https://web.expasy.org/protparam/
} 
the TransScript One-Step gDNA Removal and cDNA Synthesis SuperMix Kit (Trans, Beijing), $1.0 \mu \mathrm{g}$ of RNA was reverse transcribed to cDNAs, and the cDNAs were diluted 10 times with sterile water used as a template for quantitative realtime PCR (qRT-PCR). $\beta$-Actin (FJ618517), $\alpha$-tubulin (FJ618518) and $\beta$-tubulin (FJ618519) were selected as internal controls (Zang et al., 2015). All primers used for qRT-PCR are listed in Supplementary Table 1 . The reaction system parameters were the same as those described by Wang et al. (2019), and the relative quantitative analysis of gene expression was performed using the $2^{-\Delta \Delta \mathrm{Ct}}$ method (Livak and Schmittgen, 2001).

\section{Analysis of the Subcellular Location}

The full-length ThCOL2 coding region (without the stop codon) was ligated to the 5 ' end of green fluorescent protein (GFP) to generate a ThCOL2-GFP fusion vector, which was driven by the cauliflower mosaic virus (CaMV) 35S promoter. A 35S:GFP vector was used as the control. All primer sequences used are listed in Supplementary Table 1. The specific operation methods were the same as those described by Liu Z. Y. et al. (2020).

\section{Analysis of Transactivation Activity}

Based on the conserved domain, full-length ThCOL2 was truncated into three fragments. Specifically, the first fragment was the entire $\mathrm{N}$-terminus containing the two B-box domains, the second fragment was the middle region without any conserved domain, and the third fragment was the entire C-terminus containing the CCT domain. The full-length ThCOL2 (1-432 aa) and multiple truncated fragments, dC1 (1-138 aa), dC2 (139346 aa), dC3 (347-432 aa), dC4 = dC1 + dC2 (1-346 aa), and $\mathrm{dC} 5=\mathrm{dC} 2+\mathrm{dC} 3(139-432 \mathrm{aa})$, were fused to the GAL4 DNA binding domain in the pGBKT7 vector. All primer sequences are listed in Supplementary Table 1. The domain required for transcriptional activation was determined by screening the growth status on SD/-Trp/-His/X-a-Gal media. The specific experimental steps were performed according to the instructions of Matchmaker $^{\mathrm{TM}}$ (Gold Yeast Two-Hybrid System).

\section{Vector Construction and Transient Expression of ThCOL2 in T. hispida}

The CDS of ThCOL2 was inserted into pROKII and driven by the 35S CaMV promoter to overexpress ThCOL2 (35S:COL). An inverted repeat truncated CDS of ThCOL2 that was $246 \mathrm{bp}$ in length was inserted into pFGC5941 at the two ends of the CHSA intron (pFGC:COL) to silence ThCOL2 expression (Supplementary Figure 1). The primers used for this experiment were listed in Supplementary Table 1. The genetic transformation of $T$. hispida plants was performed according to the protocol described by Liu Z. Y. et al. (2020). Three types of transiently transformed plantlets and at least nine seedlings of each type of transgenic T. hispida plant were harvested and used for qRT-PCR analysis. Three independent experiments were performed.

\section{Biochemical Staining and Physiological Measurements of Transformed Plants}

After culture for $48 \mathrm{~h}$ on $1 / 2 \mathrm{MS}$ medium, the seedlings from transient transgenic T. hispida (OE, IE, and Con) were transferred either to fresh 1/2 MS medium or 1/2 MS supplemented with $150 \mathrm{mM} \mathrm{NaCl}$ for $2 \mathrm{~h}$. Then, the seedlings were harvested and incubated with NBT, DAB, or Evans blue solutions. The procedures for NBT and DAB staining were performed using the method reported by Zhang et al. (2011). Evans blue staining was performed as described by Kim et al. (2003). Each sample contained at least nine harvested seedlings, and three independent experiments were performed.

In addition, after culture for $48 \mathrm{~h}$ on $1 / 2 \mathrm{MS}$ medium, the plants were transferred to $1 / 2 \mathrm{MS}$ medium containing $150 \mathrm{mM} \mathrm{NaCl}$ for either 12 or $24 \mathrm{~h}$ and then harvested for physiological analyses. POD, electrolyte leakage and MDA measurements were performed using the methods reported by Zang et al. (2015). $\mathrm{H}_{2} \mathrm{O}_{2}$ levels, SOD activity and CAT activity were measured according to the instructions provided with the reagent kit (Nanjing Jiancheng Bioengineering Institute, China). Each sample contained at least 9 seedlings, and three independent experiments were performed. qRT-PCR was used to detect the relative expression levels of five ABA biosynthesis-related genes (NCED1, 3, 4, ZEP, and AAO3) (Tan et al., 2020) in OE transgenic plants after normal condition and stress with $150 \mathrm{mM}$ $\mathrm{NaCl}$ for $24 \mathrm{~h}$. The primers used for qRT-PCR are listed in Supplementary Table 1.

\section{Statistical Analysis}

The data were compared using Student's $t$-test. *Indicates a significant difference $(P<0.05)$, ** represents a very significant difference $(P<0.01)$.

\section{RESULTS}

\section{Identification of ThCOL2 Genes in T. hispida}

In total, eight candidate COL genes (named ThCOL1-ThCOL8) with complete ORFs were identified from the $T$. hispida transcriptome. The total length of the 8 ThCOL genes was 1,089$1,467 \mathrm{bp}$. The number of amino acid residues ranged from 362 to 488 , and the predicted molecular weights (MWs) were $39.6-53.6 \mathrm{kDa}$, with a theoretical isoelectric PI of $4.83-7.48$ (Table 1).

TABLE 1 | Features of ThCOL genes in Tamarix hispida.

\begin{tabular}{lccccc}
\hline Name & $\begin{array}{c}\text { GenBank accession } \\
\text { numbers }\end{array}$ & $\begin{array}{c}\text { ORF } \\
\text { (bp) }\end{array}$ & $\begin{array}{c}\text { Protein } \\
\text { length }\end{array}$ & $\begin{array}{c}\text { Theoretical } \\
\text { pl }\end{array}$ & $\begin{array}{c}\text { Molecular } \\
\text { weight (kD) }\end{array}$ \\
\hline ThCOL1 & MW846245 & 1,176 & 391 & 4.97 & 42.12 \\
ThCOL2 & MW846246 & 1,296 & 431 & 5.78 & 47.75 \\
ThCOL3 & MW846247 & 1,404 & 467 & 5.83 & 51.9 \\
ThCOL4 & MW846248 & 1,218 & 405 & 5.95 & 45.5 \\
ThCOL5 & MW846249 & 1,089 & 362 & 7.48 & 39.6 \\
ThCOL6 & MW846250 & 1,467 & 488 & 7.03 & 53.6 \\
ThCOL7 & MW846251 & 1,188 & 395 & 4.83 & 42.9 \\
ThCOL8 & MW846252 & 1,137 & 378 & 5.49 & 41.7 \\
\hline
\end{tabular}

ORF, open reading frame; bp, base pair; aa, amino acid; $M W$, molecular weight; pl, isoelectric point; $\mathrm{kDa}$, kilodalton. 


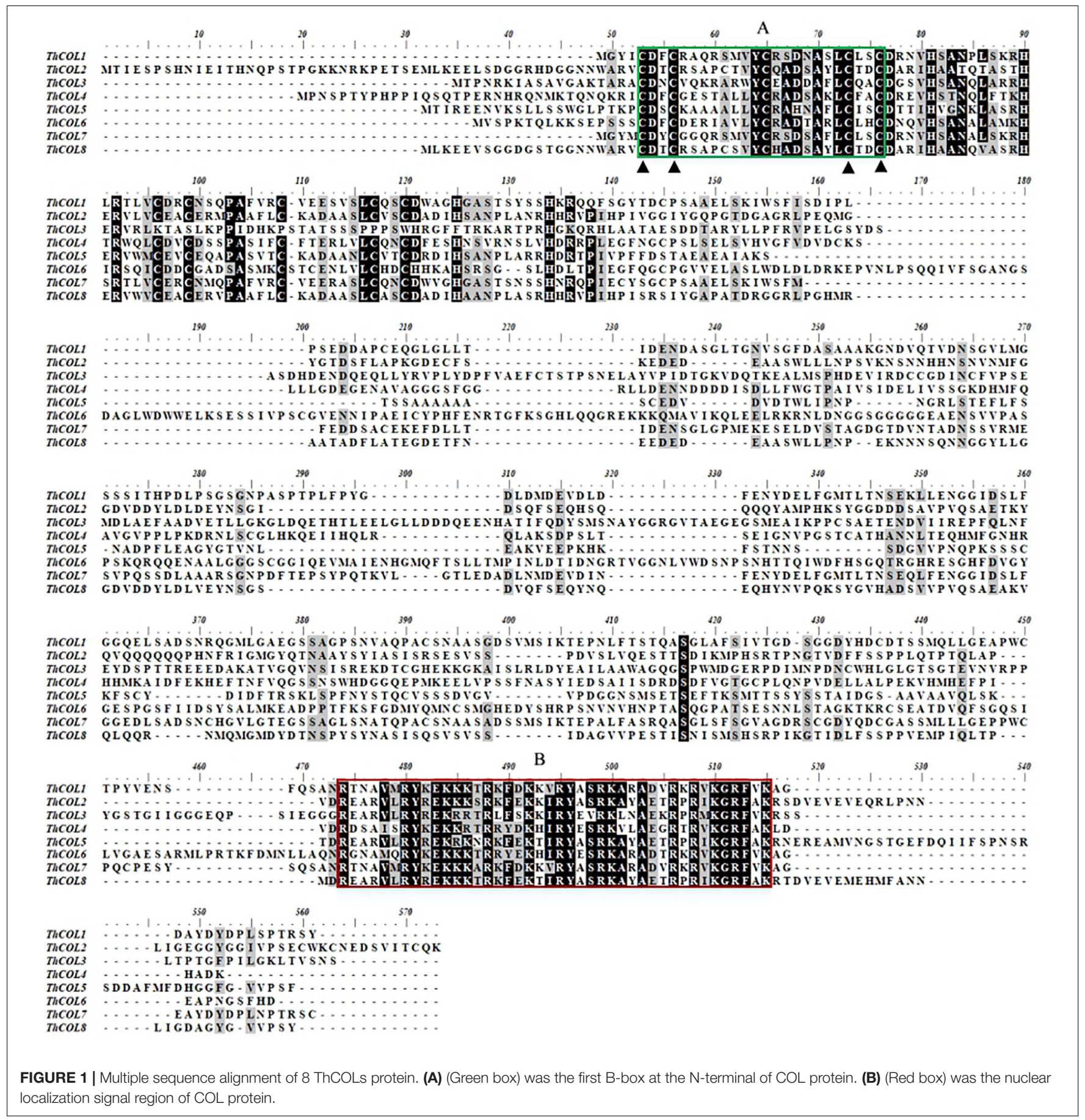

\section{Classification of Eight ThCOLs}

Multiple sequence alignments were performed on $8 \mathrm{COL}$ proteins from $T$. hispida. The amino acid identity of the eight ThCOL proteins ranged from 22.83 to $71.95 \%$. The proteins contained a conserved B-box domain at the $\mathrm{N}$-terminus and a CCT domain at the C-terminus (Figure 1). An unrooted phylogenetic tree was constructed using MEGA with full-length protein sequences of T. hispida COLs and AtCOLs (Figure 2). The 8 ThCOL proteins clustered into three groups, of which ThCOL2, ThCOL5, and ThCOL8 were closely related, belonging to the first group, and contained two complete B-box domains at the N-terminus. ThCOL3 belonged to the second group, which contained a complete B-box domain at the N-terminus. ThCOL1, ThCOL4, ThCOL6, and ThCOL7 belonged to the third group that contained a complete B-box domain and a B-box domain with secondary structure changes at the $\mathrm{N}$-terminus (Figure 2). 


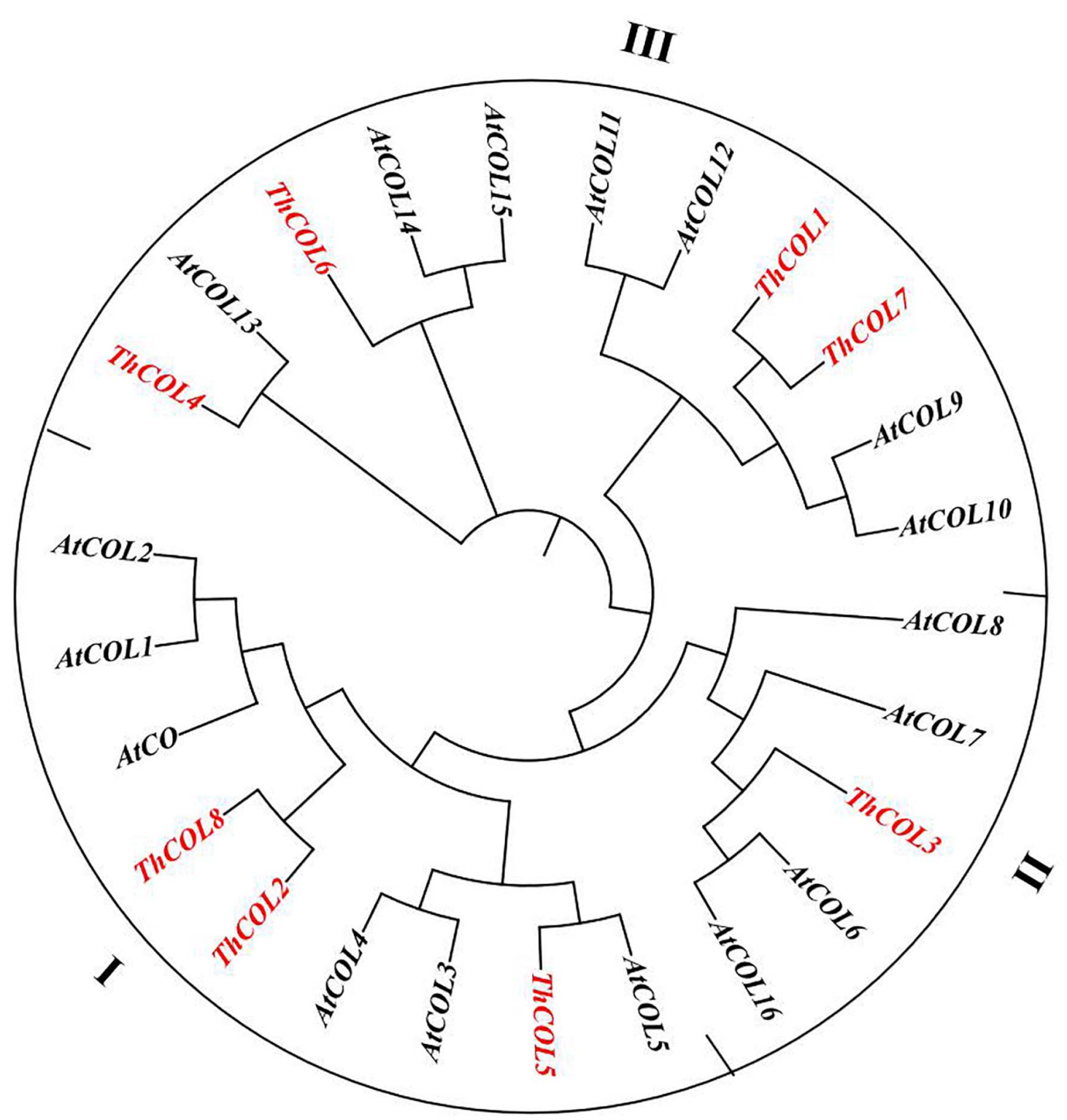

FIGURE 2 | Phylogenetic analysis of ThCOLs. Analysis was performed with both ThCOLs and Arabidopsis proteins. ThCOLs sequences were downloaded from the seven transcriptome libraries. The accession numbers of Arabidopsis sequences in the phylogenetic are: AtCO (Q39057), AtCOL1 (O50055), AtCOL2 (Q96502), AtCOL3 (Q9SK53), AtCOL4 (Q940T9), AtCOL5 (Q9FHH8), AtCOL6 (Q8LG76), AtCOL7 (Q9C9A9), AtCOL8 (Q9M9B3), AtCOL9 (Q9SSE5), AtCOL10 (Q9LUA9), AtCOL11 (O23379), AtCOL12 (Q9LJ44), AtCOL13 (O82256), AtCOL14 (O22800), AtCOL15 (Q9C7E8), and AtCOL16 (Q8RWD0).

\section{Expression Patterns of ThCOL Genes in Plants Under Several Abiotic Stresses and Treated With ABA}

Quantitative real-time PCR was used to analyze the expression patterns of ThCOL genes in plants subjected to $\mathrm{NaCl}, \mathrm{PEG}_{6,000}$, and $\mathrm{CdCl}_{2}$ stress and $\mathrm{ABA}$ treatments to investigate the functions of the ThCOL genes.

In plants under $\mathrm{NaCl}$ stress, the expression of ThCOL4, 5, 6, 7, and 8 did not change significantly in leaves. ThCOL1 and ThCOL3 were significantly upregulated at only two time points (12 and $72 ; 12-24 \mathrm{~h}$, respectively) of stress exposure. The expression of ThCOL2 was significantly upregulated at $12-72 \mathrm{~h}$ of stress exposure. In roots, the expression of ThCOL1, 2, 3, 4, and 5 was significantly upregulated at all time points of stress exposure, in which the expression of ThCOL2 peaked at $72 \mathrm{~h}$, with a value that was 6.99 times higher than the control. The expression levels of ThCOL6, 7 , and 8 at one $(12 \mathrm{~h})$, three $(6,12$, and $72 \mathrm{~h}$ ), and two time points (6 and $24 \mathrm{~h}$ ) of stress exposure, respectively, were not significantly changed, and the expression of these genes was significantly increased at other time points of stress exposure (Figure 3A).

The expression of ThCOL4, 5, 6, and 7 did not change significantly in leaves under PEG $_{6,000}$ stress (except that ThCOL5 


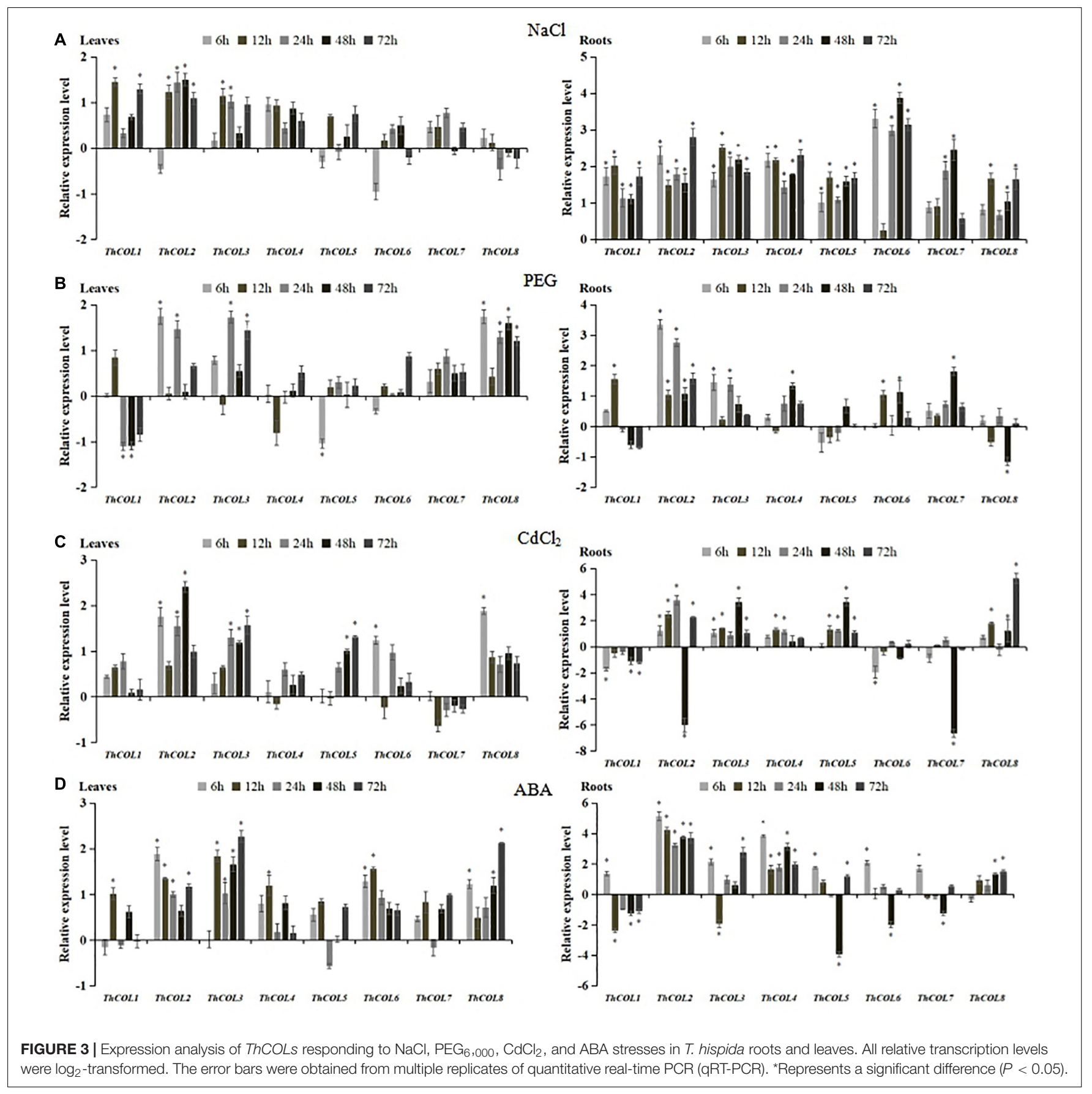

was downregulated in the early stage of stress). The expression levels of ThCOL2 and ThCOL3 were significantly upregulated at two time points (6 and 24; 24 and $72 \mathrm{~h}$, respectively) of stress exposure, but no significant changes were observed at the other time points. In contrast, the expression of ThCOL1 was significantly downregulated at two time points (24 and $48 \mathrm{~h}$ ) of stress exposure, and no significant changes were observed at other time points. ThCOL8 expression was significantly upregulated at most of all time points of stress exposure (except $12 \mathrm{~h}$ ). In roots, the expression of ThCOL1, 3, 4, 6, 7, and 8 did not change significantly at three or four time points of stress exposure. No significant change in ThCOL5 expression was observed at any time point of stress exposure. ThCOL2 expression was significantly upregulated at all time points of stress exposure, and ThCOL2 was able to produce a rapid response to $\mathrm{PEG}_{6,000}$ stress that was 10.22 times higher than the control at $6 \mathrm{~h}$ (Figure 3B).

In plants under $\mathrm{CdCl}_{2}$ stress, the expression of ThCOL1, 4 , and 7 in leaves was not significantly altered. The expression of ThCOL2 and ThCOL3 was upregulated at three time points (6, 24, and $48 ; 24-72 \mathrm{~h}$, respectively) of stress exposure. 


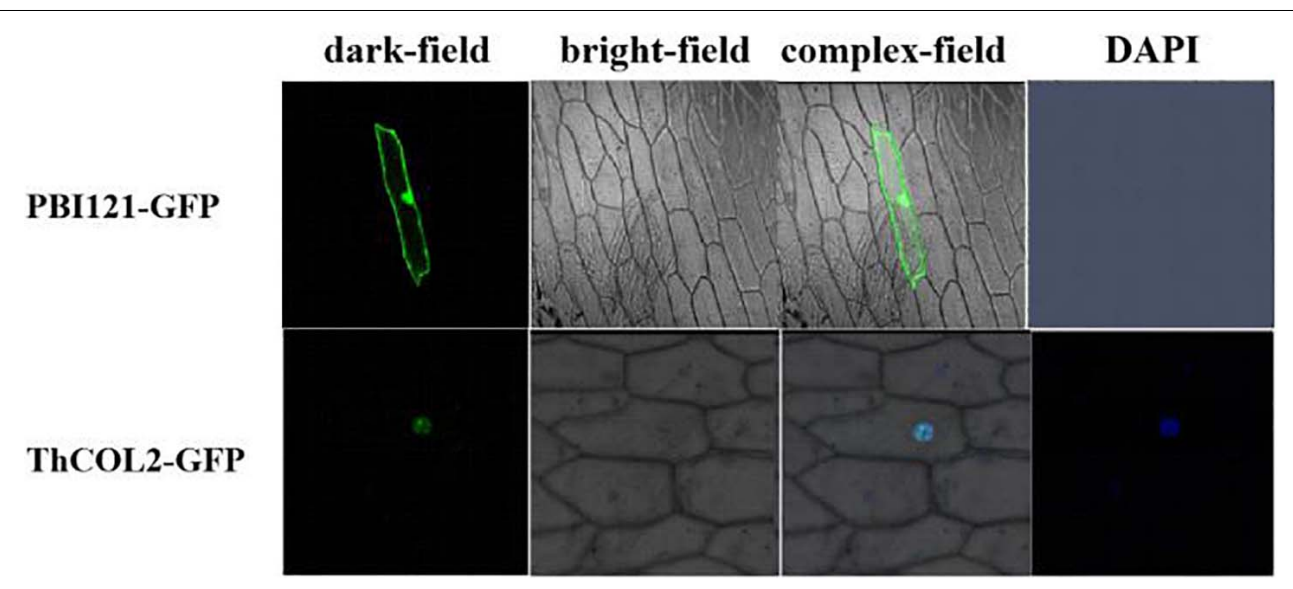

FIGURE 4 | Nuclear localization of ThCOL2 proteins. The ThCOL2-GFP fusion gene and GFP (control) were transiently expressed in onion epidermal cells by the particle bombardment (Bio-Rad, Hercules, CA, United States) method. The transformed cells were cultured on MS medium for 2-3 days and visualized using a confocal microscope at $488 \mathrm{~nm}$ for excitation of GFP and 507 nm longpass for emission (LSM410, Zeiss, Jena, Germany).

ThCOL5 expression did not change significantly in the early stage of stress but increased significantly at $48-72 \mathrm{~h}$. The expression levels of ThCOL6 and ThCOL8 were upregulated at $6 \mathrm{~h}$, but were not significantly change at $12-72 \mathrm{~h}$. In roots, the expression of ThCOL4 was significantly upregulated at 12-24 $\mathrm{h}$ of stress exposure, the expression of ThCOL6 and 7 was significantly downregulated at one time point (6 and $48 \mathrm{~h}$, respectively) of stress exposure. ThCOL 1 expression was significantly downregulated at 6 , and $48-72 \mathrm{~h}$ of stress exposure, while ThCOL3, 5, and 8 expression levels were significantly upregulated or remained unchanged at all time points. Notably, ThCOL2 expression increased gradually from 6 to $24 \mathrm{~h}$, decreased sharply at 48 h to only $1.5 \%$ of the control level, and increased significantly at $72 \mathrm{~h}$ (Figure 3C).

After the ABA treatment, the expression of ThCOL2, 3, and 8 in leaves was significantly increased or remained unchanged at all time points. The expression of ThCOL1, 4, and 6 were upregulated at $12 \mathrm{~h}$ of stress exposure. The expression of ThCOL5 and 7 did not change significantly at all time points of stress exposure. In roots, the expression of all ThCOL genes was divided into two groups with distinct expression trends. The first group included ThCOL1, 5, 6, and 7, the expression of which was significantly inhibited at $48 \mathrm{~h}$ of stress exposure, the ThCOL3 expression was significantly inhibited at $12 \mathrm{~h}$ of stress exposure. In particular, ThCOL5 expression was the most obviously inhibited gene at $48 \mathrm{~h}$, and the expression level was only $6.6 \%$ of the control. The expression of the second group (ThCOL2, 4, and 8) was significantly increased or remained unchanged at all stress time points, among which ThCOL2 and ThCOL4 potentially respond early to ABA treatment and reached peak expression at $6 \mathrm{~h}$; the expression was 35.03 and 14.44 times higher than the control, respectively (Figure 3D).

Based on these results, all 8 ThCOL genes responded to three abiotic stresses (salt, drought and heavy metals) and ABA treatment. Notably, in plants exposed to $\mathrm{PEG}_{6}, 000, \mathrm{CdCl}_{2}$, and $\mathrm{ABA}$ treatments, the expression of ThCOL2 in roots changed most significantly. The expression of ThCOL2 in the roots and leaves of $T$. hispida under $\mathrm{NaCl}$ stress was significantly upregulated at various time points. Thus, ThCOL2 may play a more important role in the process by which plants respond to abiotic stress. Therefore, the salt tolerance function and characteristics of ThCOL2 were further studied.

\section{ThCOL2 Is Localized in the Nucleus and Exhibits Transactivation Activity}

Two days after transformation, confocal laser scanning microscopy was used to examine the cells. The confocal image showed that the ThCOL2-GFP fusion protein was targeted to the nucleus (Figure 4). The results of the transactivation activity experiment showed that all fusion plasmids allowed $\mathrm{Y} 2 \mathrm{H}$ Gold cells to grow normally on SD/-Trp medium after transformation, and pGBKT7-ThCOL2 (1-432 aa), pGBKT7-dC2 (139-346 aa), pGBKT7-dC4 (1-346 aa), and pGBKT7-dC5 (139-432 aa) allowed cells to grow and show a blue color on the screening medium (SD/-Trp/-His/X-a-Gal) (Figure 5). Based on these results, ThCOL2 has transcriptional activation activity, and the transcriptional activation domain might be located at 139-346 aa. Because dC2 did not contain the complete conserved domain of the ThCOL2 protein, we speculate that the B-box and CCT domains might not play a decisive role in the transcriptional activation activity of the ThCOL 2 protein.

\section{Generation of Transient Expression of ThCOL2 in T. hispida}

The recombinant vectors pROKII-ThCOL2 and pFGC5941ThCOL2 were successfully constructed to further verify whether the ThCOL2 gene improved salt tolerance. The empty pROKII vector served as a control. The expression level of the ThCOL2 gene in three transgenic $T$. hispida species after different coculture times was analyzed using qRT-PCR to determine the success of the overexpression and silencing of the ThCOL2 gene in $T$. hispida and to determine the appropriate time point for transient transformation. Compared with the control, 


\section{$\mathrm{SD} /$-Trp SD/-Trp/-His/X}
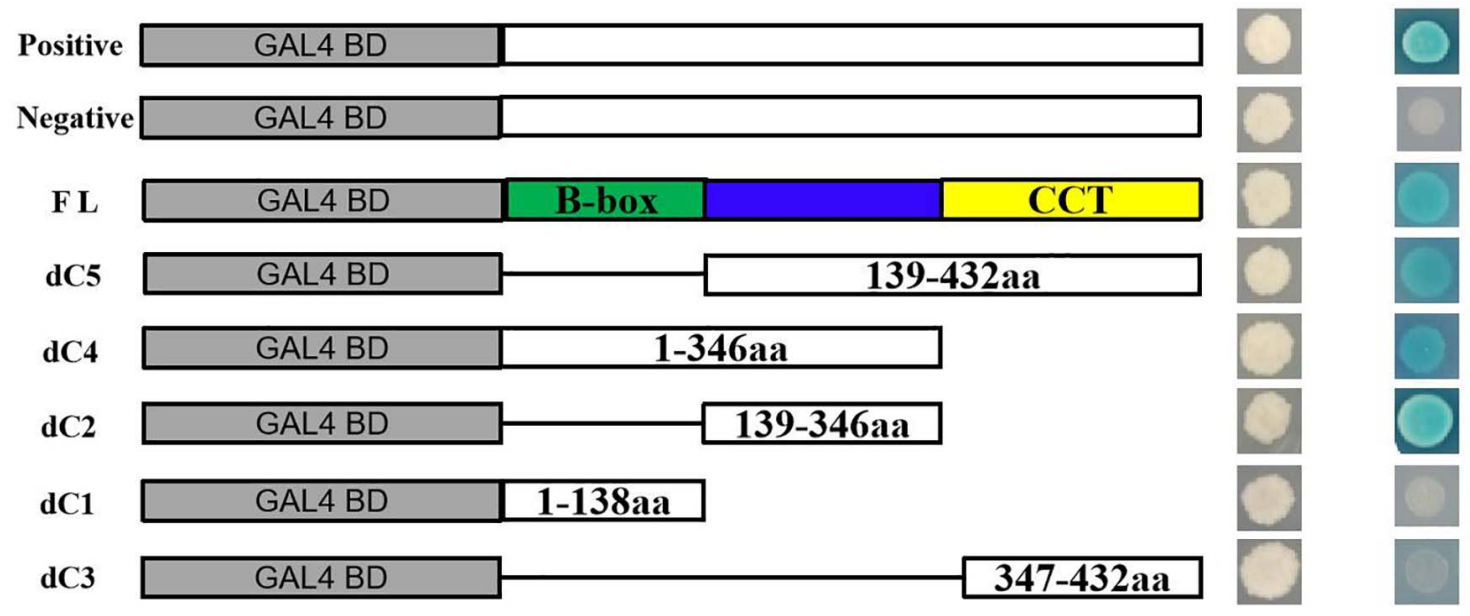

FIGURE 5 | Transcriptional activation assays of the full-length ThCOL2 protein and truncated versions lacking different protein domains. This approach uses the five-segment method to make a deletion, dividing the full-length protein into three segments $(\mathrm{dC} 1, \mathrm{dC} 2$, and $\mathrm{dC} 3)$ based on the conserved domain, while the $\mathrm{dC} 4=(\mathrm{dC} 1+\mathrm{dC} 2)$ and the $\mathrm{dC} 5=(\mathrm{dC} 2+\mathrm{dC} 3)$. This study investigates which these five fragment have transcriptional activation activity and which region was necessary for transcriptional activation. SD/-Trp and SD/-Trp/-His/X indicate SD media lacking Trp and SD media with X- $\alpha$-Gal added but lacking Trp and His, respectively. Positive control $=$ pGBKT7-53 + pGADT7-T, Negative control $=$ pGBKT7-Lam + pGADT7-T.

ThCOL2 expression was significantly increased in OE plants and significantly reduced in IE plants. In particular, at 48 and $72 \mathrm{~h}$ of cocultivation, the expression levels of ThCOL2 in OE plants were 29.8 and 4.95 times higher than the control, respectively. The expression levels of ThCOL2 in IE plants were 4.1 and $8.2 \%$ of the control, respectively (Figure 6). Thus, these plants transiently expressing ThCOL2 constructs are suitable for further research.

\section{Physiological Characterization of T. hispida With Transient Overexpression and Inhibition of ThCOL2 Expression Under Salt Stress}

The results of NBT and DAB staining showed that the coloring of the three types of transiently transformed $T$. hispida was basically the same under normal conditions. However, after $2 \mathrm{~h}$ of salt stress, the coloration of all three strains obvious increased. Among them, the IE plant was the darkest and the OE plant was the lightest, indicating that the overexpression of ThCOL2 led to a decrease in the accumulation of $\mathrm{O}^{2-}$ and $\mathrm{H}_{2} \mathrm{O}_{2}$ in the cell. The inhibition of ThCOL2 expression caused the accumulation of intracellular $\mathrm{O}^{2-}$ and $\mathrm{H}_{2} \mathrm{O}_{2}$ (Figures 7A,B). Similar results were obtained from the measurement of the $\mathrm{H}_{2} \mathrm{O}_{2}$ content. Under normal conditions, a significant difference in the $\mathrm{H}_{2} \mathrm{O}_{2}$ content was not observed among the three types of transgenic plants (CON, OE, and IE). After exposure to salt stress, the $\mathrm{H}_{2} \mathrm{O}_{2}$ content in $\mathrm{OE}$ plants was significantly less than in CON plants. The $\mathrm{H}_{2} \mathrm{O}_{2}$ content of IE plants was significantly higher than $\mathrm{CON}$ plants. Specifically, after exposure to $\mathrm{NaCl}$ stress for $12 \mathrm{~h}$, the $\mathrm{H}_{2} \mathrm{O}_{2}$ contents of $\mathrm{OE}$ and IE plants were $87 \%$ and 1.15 fold higher than CON plants, respectively. After $24 \mathrm{~h}$, the $\mathrm{H}_{2} \mathrm{O}_{2}$ contents of $\mathrm{OE}$ and IE plants were $83 \%$ and 1.24-fold higher than
CON, respectively (Figure 7D). This result suggested that the overexpression of the ThCOL2 gene significantly reduced $\mathrm{H}_{2} \mathrm{O}_{2}$ accumulation in transgenic plant cells under salt stress.

Under non-stress conditions, no significant difference in SOD activity was observed among the three types of transgenic plants. Upon exposure to salt stress, SOD activities increased in OE plants and decreased significantly in IE plants. After $12 \mathrm{~h}$ of salt stress, SOD activities in IE and OE plants were 94.6\% and 1.04fold of the values of control plants, respectively. After $24 \mathrm{~h}$ of salt stress, SOD activities in IE plants and OE plants were $97.6 \%$ and 1.02-fold the values of control plants, respectively (Figure 7E).

Under non-stress conditions, no significant difference in CAT activity was observed among the three types of transgenic plants. Upon exposure to salt stress, CAT activities increased in OE plants and decreased significantly in IE plants. After $12 \mathrm{~h}$ of salt stress, CAT activities in IE and OE plants were $82.3 \%$ and 1.07 fold, respectively, the values of control plants. After $24 \mathrm{~h}$ of salt stress, CAT activities in IE plants and OE plants were $84.3 \%$ and 1.06 -fold the values of control plants, respectively (Figure 7F).

Under non-stress conditions, no significant difference in POD activity was observed among the three types of transgenic plants. Upon exposure to salt stress, POD activities increased in OE plants and decreased significantly in IE plants. After $12 \mathrm{~h}$ of salt stress, POD activities in IE and OE plants were $77 \%$ and 1.17 -fold, respectively, the values of control plants. After $24 \mathrm{~h}$ of salt stress, POD activities in IE plants and OE plants were $75 \%$ and 1.12 fold the values of control plants, respectively (Figure 7G). Based on these results, ThCOL2 enhances ROS scavenging by increasing the activity of SOD, CAT, and POD enzymes.

Normal living cells have a complete membrane structure, preventing Evans blue from entering the cell. However, inactive cells are stained with Evans blue because of the increase in 


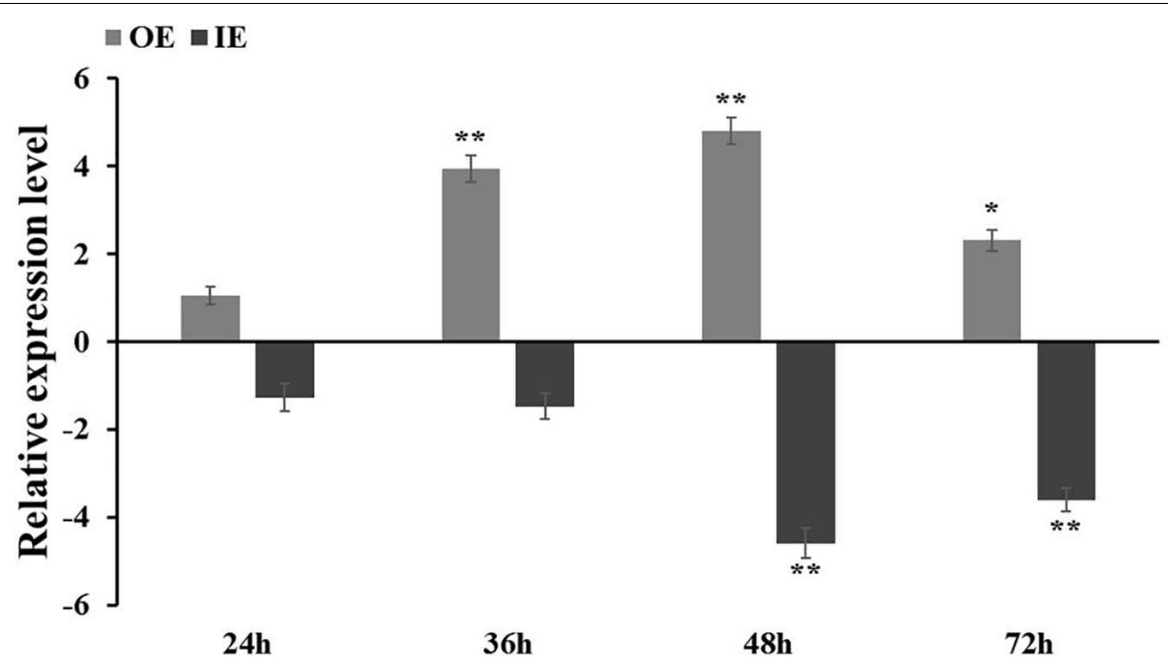

FIGURE 6 | ThCOL2 transcript levels in T. hispida plants with transient overexpression and inhibition expression. The expression data were log 2 transformed. 1-month-old T. hispida plants were transiently transformed with empty pROKII (as control), 35S:COL, pFGC:COL and were then grown on 1/2 MS medium for 24, 36, 48, and 72 h, respectively. The expression of ThCOL2 in whole OE, IE, and Con plants was measured. Error bars represent standard deviation calculated from multiple qRT-PCR replicates. OE, ThCOL2 overexpression; IE, ThCOL2 inhibition expression; Con, pROKII vector control. *Represents a significant difference $(P<0.05) .{ }^{*}$ Represents a very significant difference $(P<0.01)$.

membrane permeability. Moreover, deeper Evans blue staining is observed when more cells with no activity or an incomplete membrane are present. Therefore, Evans blue staining was used to analyze the damage to the plant cell membrane (Liu Z. Y. et al., 2020). Electrolyte leakage was used to detect the extent of cell death. In this study, Evans blue staining showed no obvious difference in the color of the three types of transgenic plants under normal conditions, but after $2 \mathrm{~h}$ of $\mathrm{NaCl}$ stress, the colors of $\mathrm{OE}$ and IE plants were obvious lighter and deeper than the control, respectively (Figure 7C). The electrolyte leakage data further confirmed these results. Under normal conditions, no obvious difference was detected between the three types of transgenic plants. After $12 \mathrm{~h}$ of salt stress, the relative conductivities of OE and IE plants were $94 \%$ and 1.05 -fold the values of Con plants, respectively. After $24 \mathrm{~h}$, the relative conductivities of $\mathrm{OE}$ and IE plants were $96 \%$ and 1.12 -fold the values of Con plants, respectively (Figure $\mathbf{7 H}$ ).

The analysis of MDA levels showed no difference between the three types of transgenic plants under normal conditions. However, upon exposure to salt stress, the MDA content of OE plants was significantly lower and the MDA content of IE plants was significantly higher than Con plants. After $12 \mathrm{~h}$ and $24 \mathrm{~h}$ of salt stress, the MDA content of $\mathrm{OE}$ plants was 89 and $88 \%$ the values of the Con plants, and the MDA content of IE plants was 1.12- and 1.08-fold the values of Con plants, respectively (Figure 7I). Thus, membrane lipid peroxidation of plants overexpressing ThCOL2 is substantially reduced.

Furthermore, the relative expression levels of five genes related to ABA biosynthesis in the OE plants were measured using qRTPCR. After $48 \mathrm{~h}$ of coculture, the expression levels of ThNCED1 and ThNCED4 were significantly higher, and the expression levels of ThNCED3, ThZEP, and ThAAO3 were not significantly altered in OE compared with CON. But after $24 \mathrm{~h}$ of salt stress, the expression levels of all five studied genes all were lower than the normal condition (Figure 7J).

\section{DISCUSSION}

Genes in the CO-like transcription factor family have been cloned and characterized in various plants, such as $17 \mathrm{COL}$ proteins in Arabidopsis (Robson et al., 2001), 16 COL proteins in rice (Yano et al., 2000), 9 COL proteins in barley (Griffiths et al., 2003), 3 COL proteins in tomato (Ben-Naim et al., 2006), and $16 \mathrm{COL}$ proteins in corn (Miller et al., 2008). In the present study, we cloned eight single ThCOL genes from $T$. hispida with complete ORFs.

CONSTANS-LIKE proteins are divided into three groups according to the B-box domains. A study on Arabidopsis COL protein function revealed that COL transcription factors with similar functions were clustered in similar types. Therefore, according to the phylogenetic grouping, the functions of the T. hispida ThCOL genes were predicted. Phylogenetic results showed that the ThCOL2, ThCOL5, and ThCOL8 genes were grouped into the first class. AtCOL4, a class I COL transcription factor, regulates tolerance to abiotic stress in an abscisic acid-dependent manner (Min et al., 2015). Steinbach (2019) showed that AtCOL4 also plays a role in suppressing flowering by functioning as a transcriptional repressor of the FT gene.

In Arabidopsis, some other COL proteins of the class I family also play roles in flowering and circadian rhythms. For example, overexpression of AtCOL5 induces flowering in Arabidopsis growing in a short day period (Hassidim et al., 2009). AtBBX32 
A

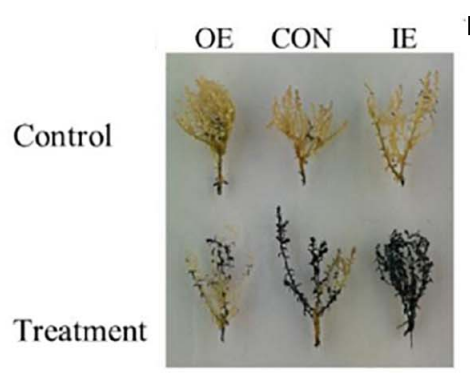

NBT

D

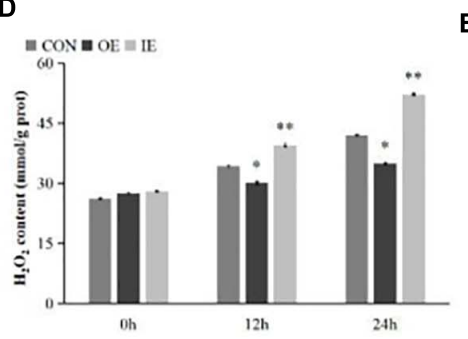

G

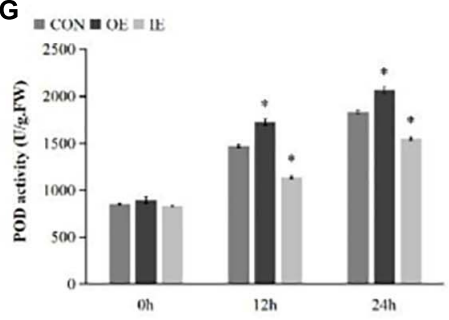

H

E $=\operatorname{CON} \approx \mathrm{OE}=\mathrm{IE}$

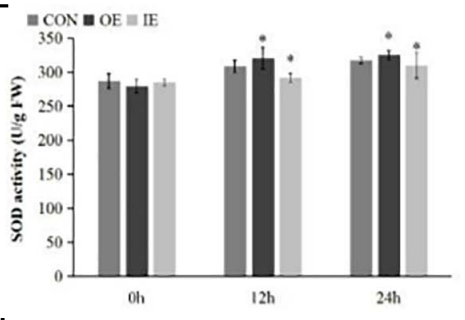

$=\operatorname{CON} \equiv \mathrm{OE}=\mathrm{IE}$

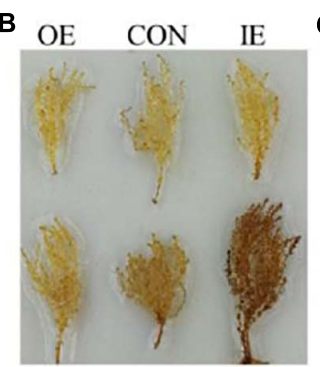

DAB

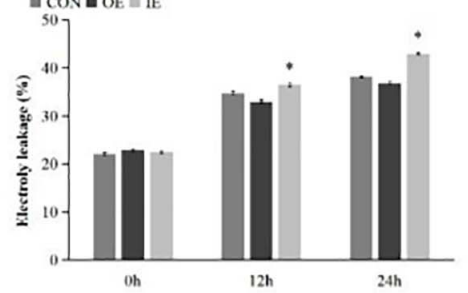

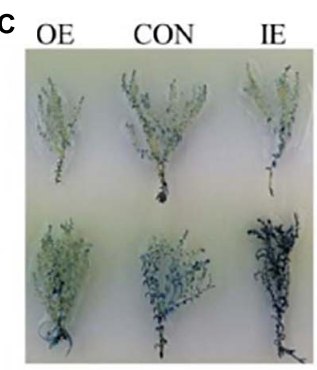

Evans blue

$\mathbf{F}$

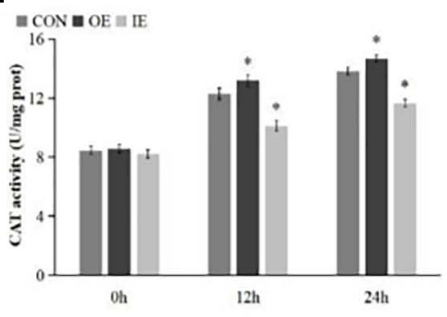

I

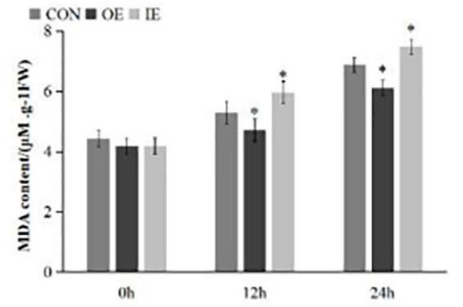

$\mathbf{J}$

0h $\square 24 h$
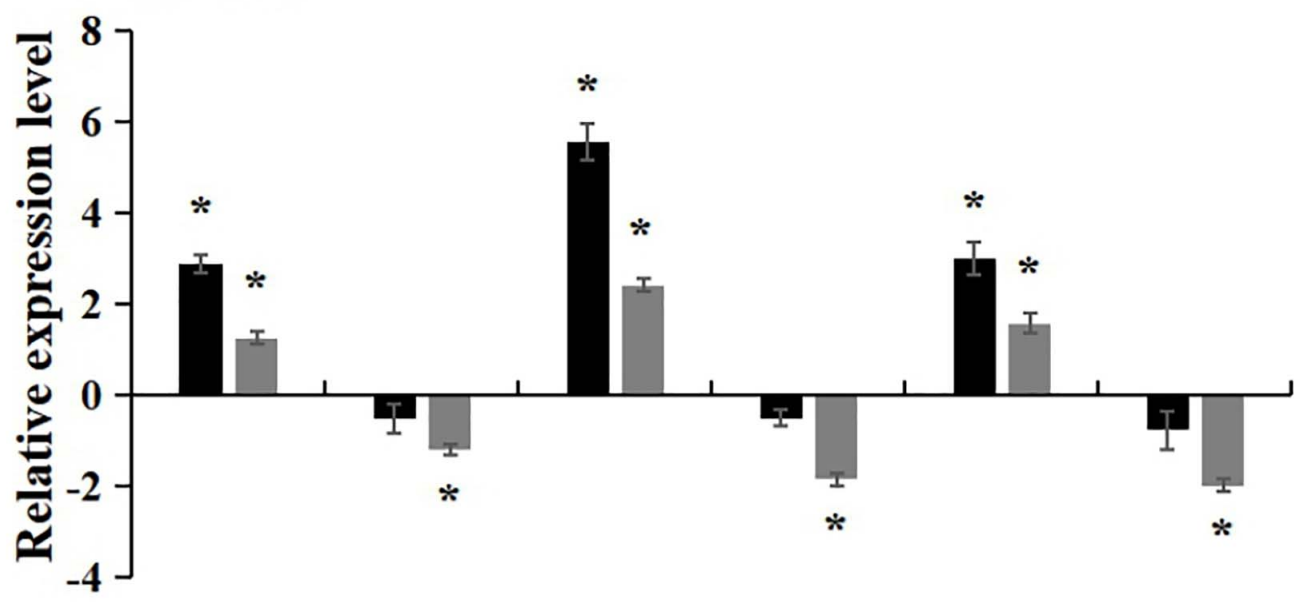

ThCOL2 ThZEP ThNCED1 ThNCED3 ThNCED4 ThAAO3

FIGURE 7 | Histochemical staining and the comparison of physiological indicators between transgenic T. hispida and control under $150 \mathrm{mM} \mathrm{NaCl} \mathrm{stress.} \mathrm{(A,B)} \mathrm{The}$ plants were stained with NBT and DAB to reveal the accumulation of $\mathrm{O}^{2-}$ and $\mathrm{H}_{2} \mathrm{O}_{2}$, respectively. (C) Evans blue staining analysis of cell death. (D) Determination of $\mathrm{H}_{2} \mathrm{O}_{2}$ contents. (E-G) SOD, CAT, and POD contents analysis the ability of cells to scavenge ROS. (H) Analysis of cell death by measurement of electrolyte leakage. (I) MDA contents analysis of OE, IE, and Con plants. ${ }^{*}$ Represents a significant difference $(P<0.05)$. ${ }^{*}$ Represents a very significant difference $(P<0.01)$. (J) Analysis of the expressions of 5 ABA biosynthesis genes in T. hispida plants with transient over-expressioned ThCOL2. "O h" represent after $48 \mathrm{~h}$ of coculture, $0 \mathrm{~h}$ of salt stress "24 h" represent after $48 \mathrm{~h}$ of coculture, $24 \mathrm{~h}$ of salt stress. The expression data were $\log _{2}$ transformed. *Represents a significant difference $(P<0.05)$. ${ }^{\star \star}$ Represents a very significant difference $(P<0.01)$. 
interacts with AtCOL3 to regulate flowering (Tripathi et al., 2017). Ledger et al. (2001) analyzed the circadian rhythms of transgenic plants overexpressing AtCOL1 and showed that overexpression of AtCOL1 can shorten two different circadian rhythm cycles. Experiments with the highest expression of AtCOL1-overexpressing lines showed that circadian deficiency depends on the fluence rate, suggesting that AtCOL1 affects the light input pathway. Therefore, we speculated that the ThCOL2, ThCOL5, and ThCOL8 genes may participate in the responses to abiotic stress and the photoperiod.

Quantitative real-time PCR was performed to analyze the expression of the $8 \mathrm{ThCOL}$ genes in plants exposed to three abiotic stresses and $\mathrm{ABA}$ treatments to determine whether the 8 ThCOL genes were involved in the stress response in T. hispida. All of these genes allowed the plants to respond to one or more of these stress treatments, and their expression patterns varied. And the obvious differential time-course expressions of these eight genes were found. For example, in leaves, upregulation of ThCOL2 gene under PEG treatment at 6 and 24, but not at 12, 48 , and $72 \mathrm{~h}$. At the time, continuously upregulation of ThCOL2 gene under $\mathrm{NaCl}$ treatment at 6-72 $\mathrm{h}$. This phenomenon maybe common in plant, such as AtCOL4 expression was significantly upregulated after $3 \mathrm{~h}$ of $\mathrm{NaCl}$ stress, and returned to the control level after $6 \mathrm{~h}$ of $\mathrm{NaCl}$ stress (Min et al., 2015). While BnCOL2 expression continued to decrease after 1,3 , and $6 \mathrm{~h}$ of $\mathrm{NaCl}$ stress in Brassica napus (Liu L. D. et al., 2020). We speculate that the expression of some genes may be expressed regularly throughout the time, but some genes have relatively large differences at different time points.

In particular, the expression of the ThCOL2 gene in the roots and stems of $T$. hispida changed significantly at most time points of exposure to the three abiotic stresses (salt, drought and heavy metals) and ABA treatments. Thus, it may play an important role in the ability of T. hispida to resist stress. Especially, under salt stress, ThCOL2 gene was significantly upregulated at all time points in leaves and roots (except $6 \mathrm{~h}$ in leaves).

In this study, both ThCOL2 and AtCOL4 are class I COL proteins. Min et al. (2015) showed that mutation of the atcol4 gene in Arabidopsis resulted in increased sensitivity to $\mathrm{ABA}$ and salt stress during seed germination and cotyledon regeneration, while overexpression of the AtCOL4 gene reduced plant sensitivity to ABA and salt stress. Moreover, in AtCOL4overexpressing and wild-type plants under ABA or salt stress, the transcript levels of other ABA biosynthesis- and stressrelated genes were increased. Based on these results, AtCOL4 participates in $\mathrm{ABA}$ and salt stress responses through $\mathrm{ABA}$ dependent signaling pathways.

The qRT-PCR results revealed significantly increased expression of ThCOL2 in the roots and stems of T. hispida under $\mathrm{NaCl}$ and $\mathrm{ABA}$ stress at most time points. Given this consistent trend, we speculate that ThCOL2 is also involved in the ABA pathway-related response to $\mathrm{NaCl}$ stress. The relative expression levels of the genes related to ABA biosynthesis in the ThCOL2 OE transgenic lines were measured using qRT-PCR, and the results indicated that the ThCOL2 gene might regulate the expression of some ABA biosynthesis genes. These findings will be further verified in future studies.
Tamarix hispida is a woody halophyte with a very high salt tolerance. Therefore, the salt tolerance function of the ThCOL2 gene was preliminarily and quickly identified through transient transformation. Histochemical staining and physiological index measurements showed that overexpression of ThCOL2 enhanced the salt tolerance of transgenic plants by increasing the activity of protective enzymes, reducing the accumulation of ROS and MDA in plants, and reducing cell damage. Interestingly, OMG1, a Arabidopsis class III COL protein, has played a role in regulating ROS pathway function by regulating the expression of genes involved in the ROS pathway (MYB77 and GRX480) (Sng et al., 2018). ThCOL2 overexpression also regulates the accumulation of ROS in plants to reduce damage caused by salt stress, indicating that class I and III COL proteins all play a certain role in regulating ROS pathway function. Whether the temporal expression changes of ThCOL2 in response to salt stress affects its physiological regulation function, and the relationship between them will further analysis through stable transform ThCOL2 into T. hispida.

The CCT domain of the COL protein is involved in nuclear localization and protein-protein interactions (Kurup et al., 2000). In this study, the ThCOL2 protein was localized in the nucleus. Moreover, the ThCOL2 protein exhibited transcriptional activation activity. Therefore, the ThCOL2 protein has the general characteristics of a transcription factor. An analysis of the transcriptional activation domain of the ThCOL2 protein revealed that the transcriptional activation domain of the protein is located in the middle segment between the B-box and CCT domains, consistent with the results of a previous study by Min et al. (2015).

In summary, 8 ThCOL genes were cloned from T. hispida in this study, and the expression patterns of these genes in response to various stresses were analyzed. The salt tolerance function of ThCOL2 was further studied by transient transformation, and the salt tolerance mechanism and protein characteristics were preliminarily analyzed. In subsequent studies, we will further transform ThCOL2 into T. hispida or other model plants and analyze the salt tolerance function and its regulatory mechanism in transgenic plants.

\section{CONCLUSION}

The CO-like transcription factor is a key transcription factor that plays an important role in regulating plant flowering. In addition, it is also involved in the abiotic stress response of plants. In this study, 8 ThCOL genes were identified in T. hispida. ThCOL gene expression was obviously altered in response to abiotic stress (salt, osmotic and heavy metal stress) and ABA treatment. Further experiments performed using the ThCOL2 gene, which responded significantly to salt stress. ThCOL2 is located in the nucleus and has transcriptional activation activity. The transcriptional activation domain is located at 139-346 aa. Moreover, overexpression of ThCOL2 in T. hispida under salt stress not only enhanced the ROS scavenging capacity to reduce cell damage and death caused by salt stress but also increased the activity of protective enzymes. 
Conversely, transiently transformed plants with a construct that inhibited ThCOL2 expression displayed the opposite physiological changes. Thus, ThCOL2 effectively enhances the tolerance of transgenic T. hispida to salt stress.

\section{DATA AVAILABILITY STATEMENT}

The datasets presented in this study can be found in online repositories. The names of the repository/repositories and accession number(s) can be found in the article/Supplementary Material.

\section{AUTHOR CONTRIBUTIONS}

$\mathrm{XL}$ and BT wrote the manuscript and performed some of the assays. JL and ZL performed the assays. JW performed the data analysis. CG provided funds for the current study, designed the study, and revised the manuscript.

\section{REFERENCES}

Ben-Naim, O., Eshed, R., Parnis, A., Teper-Bamnolker, P., Shalit, A., Coupland, G., et al. (2006). The CCAAT binding factor can mediate interactions between constans-like proteins and DNA[J]. Plant J. 46, 462-476. doi: 10.1111/j.1365313X.2006.02706.X

Chen, J., Chen, J. Y., Wang, J. N., Kuang, J. F., Shan, W., and Lu, W. J. (2012). Molecular characterization and expression profiles of MaCOL1, a Constanslike gene in banana fruit. Gene 496, 110-117. doi: 10.1016/j.gene.2012. 01.008

Gao, C., Jiang, B., Wang, Y. C., Liu, G. F., and Yang, C. P. (2012). Overexpression of a heat shock protein (ThHSP18.3) from Tamarix hispida confers stress tolerance to yeast. Mol. Biol. Rep. 39, 4889-4897. doi: 10.1007/s11033-011-1284-2

Griffiths, S., Dunford, R. P., Coupland, G., and Laurie, D. A. (2003). The evolution of Constans-like gene families in barley, rice, and arabidopsis[J]. Plant Physiol. 131, 1855-1867. doi: 10.1104/pp.102.016188

Hassidim, M., Harir, Y., Yakir, E., Kron, I., and Green, R. M. (2009). Overexpression of Constans-Like 5 can induce flowering in short-day grown Arabidopsis. Planta 230, 481-491. doi: 10.1007/s00425-009-0958-7

Kim, M., Ahn, J. W., Jin, U. H., Choi, D., Paek, K. H., and Pai, H. S. (2003). Activation of the programmed cell death pathway by inhibition of proteasome function in plants. J. Biol. Chem. 278, 19406-19415. doi: 10.1074/ jbc.M210539200

Kurup, S., Jones, H. D., and Holdsworth, M. J. (2000). Interactions of the developmental regulator $\mathrm{ABI} 3$ with proteins identified from developing Arabidopsis seeds[J]. Plant J. 21, 143-155. doi: 10.1046/j.1365-313x.2000. 00663.x

Ledger, S., Strayer, C., Ashton, F., Kay, S. A., and Putterill, J. (2001). Analys is of the function of two circadian-regulated Constans-Like genes. Plant J. 26, 15-22. doi: 10.1046/j.1365-313x.2001.01003.x

Liu, H., Dong, S. Y., Sun, D. Y., Liu, W., Gu, F. W., Liu, Y. Z., et al. (2016c). Constans-Like 9 (OsCOL9) interacts with receptor for activated C-Kinase 1(OsRACK1) to regulate blast resistance through salicylic acid and ethylene signaling pathways. PLoS One 11:e0166249. doi: 10.1371/journal.pone.0166249

Liu, H., Gu, F. W., Dong, S. Y., Liu, W., Wang, H., Chen, Z. Q., et al. (2016a). Constans-like 9 (COL9) delays the flowering time in Oryza sativa by repressing the Ehd1 pathway. Biochem. Biophys. Res. Commun. 479, 173-178. doi: 10.1016/ j.bbrc.2016.09.013

Liu, J. H., Shen, J. Q., Xu, Y., Li, X. H., Xiao, J. H., and Xiong, L. Z. (2016b). Ghd2, a Constans-like gene, confers drought sensitivity through regulation of senescence in rice. J. Exp. Bot. 67, 5785-5798. doi: 10.1093/jxb/erw344
All authors contributed to the article and approved the submitted version.

\section{FUNDING}

This work was supported by the Fundamental Research Funds for the Central Universities (No. 2572019CG01), Heilongjiang Touyan Innovation Team Program (Tree Genetics and Breeding Innovation Team) and the Overseas Expertise Introduction Project for Discipline Innovation (B16010).

\section{SUPPLEMENTARY MATERIAL}

The Supplementary Material for this article can be found online at: https://www.frontiersin.org/articles/10.3389/fpls.2021. 653791/full\#supplementary-material

\section{Supplementary Figure 1 | Construction of pFGC5941-ThCOL2 vector.}

Liu, L. D., Ding, Q. Y., Liu, J., Yang, C. L., Chen, H., Zhang, S. F., et al. (2020). Brassica napus COL transcription factor BnCOL2 negatively affects the tolerance of transgenic Arabidopsis to drought stress[J]. Environ. Exp. Bot. 178:104171. doi: 10.1016/j.envexpbot.2020.104171

Liu, Z. Y., Lei, X. J., Wang, P. L., Wang, Y. Y., Lv, J. X., Li, X. P., et al. (2020). Overexpression of ThSAP30BP from Tamarix hispida improves salt tolerance. Plant Physiol. Biochem. 146, 124-132. doi: 10.1016/j.plaphy.2019.11.020

Livak, K. J., and Schmittgen, T. D. (2001). Analysis of relative gene expression data using real-time quantitative PCR and the 2- $\Delta \Delta$ CT method. Methods 25, 402-408. doi: 10.1006/meth.2001.1262

Miller, T. A., Muslin, E. H., and Dorweiler, J. E. (2008). A maize Constans-like gene, conz1, exhibits distinct diurnal expression patterns in varied photoperiods[J]. Planta 227, 1377-1388. doi: 10.1007/s00425-008-0709-1

Min, J. H., Chung, J. S., Lee, K. H., and Kim, C. S. (2015). The Constans-like 4 transcription factor, AtCOL4, positively regulates abiotic stress tolerance through an abscisic acid-dependent manner in Arabidopsis. J. Integr. Plant Biol. 57, 313-324. doi: 10.1111/jipb.12246

Morita, R., Sugino, M., Hatanaka, T., Misoo, S. J., and Fukayama, H. (2015). CO2-responsive Constans, Constans-like, and time of chlorophyll a/b binding protein expression1 protein is a positive regulator of starch synthesis in vegetative organs of rice. Plant Physiol. 167, 1321-1331. doi: 10.1104/pp.15. 00021

Muntha, S. T., Zhang, L. L., Zhou, Y. F., Zhao, X., Hu, Z. Y., Yang, J. H., et al. (2019). Phytochrome a signal transduction 1 and Constans-Like 13 coordinately orchestrate shoot branching and flowering in leafy Brassica juncea. Plant Biotechnol. J. 17, 1333-1343. doi: 10.1111/pbi.13057

Plunkett, B. J., Henry-Kirk, R., Friend, A., Diack, R., Helbig, S., Mouhu, K., et al. (2019). Apple B-box factors regulate light-responsive anthocyanin biosynthesis genes. Sci. Rep. 9:17762. doi: 10.1038/s41598-019-54166-2

Putterill, J., Robson, F., Lee, K., Simon, R., and Coupland, G. (1995). The CONSTANS gene of Arabidopsis promotes flowering and encodes a protein showering similarities to zinc finger transcription factors. Cell 80, 847-857. doi: 10.1016/0092-8674(95)90288-0

Robson, F., Costa, M. M. R., Hepworth, S. R., Vizir, I., Pineiro, M., Reeves, P. H., et al. (2001). Functional importance of conserved domains in the floweringtime gene Constans demonstrated by analysis of mutant alleles and transgenic plants. Plant J. 28, 619-631. doi: 10.1046/j.1365-313x.2001.01163.x

Sheng, P. K., Wu, F. Q., Tan, J. J., Zhang, H., Ma, W. W., Chen, L. P., et al. (2016). A constans-like transcriptional activator, OsCOL13, functions as a negative regulator of flowering downstream of OsphyB and upstream of Ehd1 in rice. Plant Mol. Biol. 92, 209-222. doi: 10.1007/s11103-016-0506-3 
Sng, N. J., Kolaczkowski, B., Ferl, R. J., and Paul, A. L. (2018). A member of the Constans-Like protein family is a putative regulator of reactive oxygen species homeostasis and spaceflight physiological adaptation. AoB Plants 11:ly075. doi: 10.1093/aobpla/ply075

Song, N. N., Xu, Z. L., Wang, J., Qin, Q. Q., Jiang, H. Y., Si, W. N., et al. (2018). Genome-wide analysis of maize Constans-Like gene family and expression profiling under light/dark and abscisic acid treatment. Gene 673, 1-11. doi: 10.1016/j.gene.2018.06.032

Steinbach, Y. (2019). The Arabidopsis thaliana constans-like 4 (COL4)a modulator of flowering time. Front. Plant Sci. 10:651. doi: 10.3389/fpls.2019.00651

Tamura, K., Peterson, D., Peterson, N., Stecher, G., Nei, M., and Kumar, S. (2011). MEGA5: molecular evolutionary genetics analysis using maximum likelihood, evolutionary distance, and maximum parsimony methods. Mol. Biol. Evol. 28, 2731-2739. doi: 10.1093/molbev/msr121

Tan, J. J., Jin, M. N., Wang, J. C., Wu, F. Q., Sheng, P. K., Cheng, Z. J., et al. (2016). OsCOL10, a constans-like gene, functions as a flowering time repressor downstream of Ghd7 in rice. Plant Cell Physiol. 57, 798-812. doi: 10.1093/pcp/ pcw025

Tan, J. J., Wu, F. Q., and Wan, J. M. (2017). Flowering time regulation by the constans-like gene OsCOL10. Plant Signal. Behav. 12:e1267893. doi: 10.1080/ 15592324.2016.1267893

Tan, Z. L., Wen, X. J., and Wang, Y. C. (2020). Betula platyphylla BpHOX2 transcription factor binds to different cis-acting elements and confers osmotic tolerance. J. Integr. Plant Biol. 62, 1762-1779. doi: 10.1111/jipb. 12994

Tripathi, P., Carvallo, M., Hamilton, E. E., Preuss, S., and Kay, S. A. (2017). Arabidopsis B-box32 interacts with constans-like3 to regulate flowering. Proc. Natl. Acad. Sci. U.S.A. 114, 172-177. doi: 10.1073/pnas.1616459114

Wang, H. G., Zhang, Z. L., Li, H. Y., Zhao, X. Y., Liu, X. M., Ortiz, M., et al. (2013). Constans-like 7 regulates branching and shade avoidance response in Arabidopsis. J. Exp. Bot. 64, 1017-1024. doi: 10.1093/jxb/ers376

Wang, L., Wang, C., Wang, D., and Wang, Y. (2014). Molecular characterization and transcript profiling of NAC genes in response to abiotic stress in Tamarix hispida. Tree Genet. Geno. 10, 157-171. doi: 10.1007/s11295-0130672-2

Wang, P. L., Wang, L. Q., Liu, Z. Y., Zhang, T. Q., Wang, Y. Y., Li, Y. B., et al. (2019). Molecular characterization and expression profiles of GRAS genes in response to abiotic stress and hormone treatment in Tamarix hispida[J]. Trees 33, 213-225. doi: 10.1007/s00468-018-1771-3

Wu, W. X., Zhang, Y. X., Zhang, M., Zhan, X. D., Shen, X. H., Yu, P., et al. (2018). The rice constans-like protein OsCOL15 suppresses flowering by promoting Ghd7 and repressing RID1. Biochem. Biophys. Res. Commun. 495, 1349-1355. doi: 10.1016/j.bbrc.2017.11.095

Xiao, G. H., Li, B. J., Chen, H. J., Chen, W., Wang, Z. Y., Mao, B. Z., et al. (2018). Overexpression of $\mathrm{P} v \mathrm{CO} 1$, a bamboo Constans-like gene, delays flowering by reducing expression of the FT gene in transgenic Arabidopsis. BMC Plant Biol. 18:232. doi: 10.1186/s12870-018-1469-0

Yano, M., Katayose, Y., Ashikari, M., Yamanouchi, U., Monna, L., Fuse, T., et al. (2000). Hd1, a major photoperiod sensitivity quantitative trait locus in rice, is closely related to the Arabidopsis flowering time gene Constans[J]. Plant Cell. 12, 2473-2483. doi: 10.1105/tpc.12.12.2473

Zang, D., Wang, C., Ji, X., and Wang, Y. (2015). Tamarix hispida zinc finger protein ThZFP1 participates in salt and osmotic stress tolerance by increasing proline content and SOD and POD activities. Plant Sci. 235, 111-121. doi: 10.1016/j.plantsci.2015.02.016

Zhang, X., Wang, L., Meng, H., Wen, H., Fan, Y., and Zhao, J. (2011). Maize ABP9 enhances tolerance to multiple stresses in transgenic Arabidopsis by modulating ABA signaling and cellular levels of reactive oxygen species. Plant Mol. Biol. 75, 365-378. doi: 10.1007/s11103-011-9732-x

Zhang, Z. L., Ji, R. H., Li, H. Y., Zhao, T., Liu, J., Lin, C. T., et al. (2014). Constanslike 7 (COL7) is involved in phytochrome B (phyB)-mediated light-quality regulation of auxin homeostasis. Mol. Plant 7, 1429-1440. doi: 10.1093/mp/ ssu058

Conflict of Interest: The authors declare that the research was conducted in the absence of any commercial or financial relationships that could be construed as a potential conflict of interest.

Copyright (C) 2021 Lei, Tan, Liu, Wu, Lv and Gao. This is an open-access article distributed under the terms of the Creative Commons Attribution License (CC BY). The use, distribution or reproduction in other forums is permitted, provided the original author(s) and the copyright owner(s) are credited and that the original publication in this journal is cited, in accordance with accepted academic practice. No use, distribution or reproduction is permitted which does not comply with these terms. 